\title{
Analysis of Different Uncertainty Activation Cross Section Data Libraries for LWR, ADS and DEMO Neutron Spectra
}

\author{
C.J. Díez ${ }^{1}$, O. Cabellos ${ }^{1,2}$ and J.S. Martínez ${ }^{1}$ \\ ${ }^{1}$ Nuclear Engineering Department, Universidad Politécnica de Madrid, Madrid, Spain \\ ${ }^{2}$ Nuclear Fusion Institute, Universidad Politénica de Madrid, Madrid, Spain
}

\begin{abstract}
This work is aimed to present the main differences of nuclear data uncertainties among three different nuclear data libraries: EAF-2007, EAF-2010 and SCALE-6.0, under different neutron spectra: LWR, ADS and DEMO (fusion). To take into account the neutron spectrum, the uncertainty data are collapsed to onegroup. That is a simple way to see the differences among libraries for one application. Also, the neutron spectrum effect on different applications can be observed. These comparisons are presented only for (n,fission), $\left(\mathrm{n}\right.$, gamma) and $(\mathrm{n}, \mathrm{p})$ reactions, for the main transuranic isotopes $\left({ }^{234,235,236,238} \mathrm{U},{ }^{237} \mathrm{~Np},{ }^{238,239,240,241} \mathrm{Pu}\right.$, $\left.{ }^{241,242 \mathrm{~m}, 243} \mathrm{Am},{ }^{242,243,244,245,246,247,248} \mathrm{Cm},{ }^{249} \mathrm{Bk},{ }^{249,250,251,252} \mathrm{Cf}\right)$. But also general comparisons among libraries are presented taking into account all included isotopes. In other works, target accuracies are presented for nuclear data uncertainties; here, these targets are compared with uncertainties on the above libraries. The main results of these comparisons are that EAF-2010 has reduced their uncertainties for many isotopes from EAF-2007 for (n,gamma) and (n,fission) but not for (n,p); SCALE-6.0 gives lower uncertainties for (n,fission) reactions for ADS and PWR applications, but gives higher uncertainties for $(n, p)$ reactions in all applications. For the (n,gamma) reaction, the amount of isotopes which have higher uncertainties is quite similar to the amount of isotopes which have lower uncertainties when SCALE-6.0 and EAF-2010 are compared. When the effect of neutron spectra is analysed, the ADS neutron spectrum obtained the highest uncertainties for (n,gamma) and (n,fission) reactions of all libraries.
\end{abstract}

\section{Introduction}

Nowadays, the knowledge of uncertainty in criticality, burn-up and depletion calculations due to cross-section data is a critical issue because of the safety and economical performance of nuclear power.

These uncertainty data are available in few nuclear data libraries, but these values are different among libraries. Then, depending on which application and which library is used, different final uncertainties will be obtained after studies of uncertainty propagation. In this work, activation nuclear data libraries and general purpose nuclear data library are compared: EAF-2007, EAF-2010 and SCALE-6.0.

General differences among these libraries are presented taking into account all included isotopes. To condense this large amount of information, one library is taken as reference (EAF-2010), and collapsed values of other libraries are divided by the reference.

Different works were performed in order to point out which nuclear data should be improved for different future nuclear applications, setting target accuracies for PWR [1] and ADS [2]. Using this information, main transuranic isotopes and (n,gamma), (n,fission) and (n,p) reactions are chosen for being studied here. Also, PWR and ADS neutron spectra are used to analyse their effect. As fusion applications are in the development stage, DEMO application is chosen as other application to compare the uncertainty libraries by using the associated spectrum and by analysing its neutron spectrum effect on uncertainties.

To compare uncertainty values, they should first be collapsed into one-group. The main reason is to take an overview of the uncertainty value without taking into account the energy structure, because each library has its own way of presenting uncertainties, not only concerning the energy structure but also the format. Also, it simplifies the general comparison among libraries taking into account all nuclides.

The purpose of this work is to show the differences between uncertainties of different nuclear data libraries and the effect of different neutron spectra. 


\section{Resume of Nuclear Data Libraries}

\subsection{EAF-2007 and EAF-2007/UN}

The activation-oriented nuclear data library EAF-2007 (European Activation File) [3] is a collection of nuclear data aimed at nuclide inventory calculations due to neutron or charged particle activation. One of its components is a cross section data library for neutrons, which comprises cross sections from low energies $\left(10^{-5}\right.$ $\mathrm{eV})$ to high energies $(60 \mathrm{MeV})$ for nuclides from hydrogen $\left({ }^{1} \mathrm{H}_{1}\right)$ to fermium $\left({ }^{257} \mathrm{Fm}_{100}\right)$. There are 816 possible isotopes, including ground states and isomeric states, so that there are in total 65,565 reaction channels available. This library is in ENDF-6 format.

In the EAF-2007/UN library, there is information about uncertainties related to the cross sections in EAF- 2007 library. This library is in ENDF-6 format, and the tape where cross section uncertainty is found is $\mathrm{MF}=33$.

The structure of the information is as follows:

- Threshold reactions: Two energy groups, one from energy threshold to $20 \mathrm{MeV}$ and another from $20 \mathrm{MeV}$ to $60 \mathrm{MeV}$.

- Non-Threshold reactions: Four energy groups, the first one from $10^{-5} \mathrm{eV}$ to $\mathrm{Ev}$ (the end of thermal region), the second one from Ev to $\mathrm{EH}$ (the end of resonance region), the third one from $\mathrm{EH}$ to $20 \mathrm{MeV}$, and the last one from $20 \mathrm{MeV}$ to $60 \mathrm{MeV}$.

\subsection{EAF-2010 and EAF-2010/UN}

EAF-2010 is the latest release of the European Activation File [4] with the same amount of targets, 816, but an increased number of excitation functions up to 66,256. It has also gained considerably in quality and completeness. The wide range of available integral data has been used to improve the library. It ranges from low energies $\left(10^{-5} \mathrm{eV}\right)$ to high energies $(60 \mathrm{MeV})$ for nuclides from hydrogen $\left({ }^{1} \mathrm{H}_{1}\right)$ to fermium $\left({ }^{257} \mathrm{Fm}_{100}\right)$.

EAF-2010/UN is the uncertainty library, which has the same energy structure as EAF-2007/UN. In this new library, the experimental information, information from TALYS model calculations and SCAS analysis has been included as much as possible.

In cases where no experimental information is available, the uncertainty is, as in the previous EAF$2007 / \mathrm{UN}$, extracted from systematics, results of graphical information or from estimates. The results have shown that the error factors adopted in EAF-2007/UN were - for some reactions - unnecessarily conservative and new more realistic - values are proposed for important major reactions.

Recently, an EAF-2010 nuclear data library was released in ENDF-6 format for 816 isotopes with a MF33 file, where the uncertainty information are. Previously, this file was not included. This release of the EAF library is not used in this work.

\subsection{SCALE-6.0 and SCALE NUCLEAR DATA COVARIANCE LIBRARY}

The data of this library [5] come from a variety of sources, including high-fidelity covariance evaluations from ENDF/B-VII, ENDF/B-VI and JENDL-3.3, as well as approximate uncertainties obtained from a collaborative project performed by BNL, LANL and ORNL (USA).

It provides covariance information for a total of 401 materials in the 44-group structure. In general, the SCALE covariance library should be viewed as a bestestimate assessment of data uncertainties, and also includes cross correlations between reactions.

This library is based on several different uncertainty approximations with varying degrees of "fidelity" to the actual nuclear data evaluation. There are two types of uncertainty information:

-High-fidelity covariances: These covariances come from the evaluated nuclear data libraries mentioned above, where uncertainty information related to ${ }^{233 ; 235 ; 238} \mathrm{U}$; 239;240;241;242 $\mathrm{Pu} ;{ }^{241} \mathrm{Am}$ can be found.

-Low-fidelity covariances: These covariances are defined to be those that are estimated independently of a specific data evaluation. They come from a project run by BNL, LANL and ORNL, where ORNL used uncertainties in integral experiment measurements of thermal crosssections, resonance integrals and potential cross-sections to approximate the standard deviations of capture, fission and elastic scattering reactions for the thermal $(<0.5 \mathrm{eV})$ and resonance ranges $(0.5 \mathrm{eV}-5 \mathrm{keV})$, and full energy correlation was assumed for the covariances within each of these respective ranges. BNL and LANL provided estimates in the fast energy range from $5 \mathrm{keV}-20 \mathrm{MeV}$ for covariances of capture, fission, elastic, inelastic, $(\mathrm{n}, 2 \mathrm{n})$ cross-sections and prompt nubar, using optical models and empirical estimates of nuclear reaction models. The uncertainty data of the previous project is approximated by different approaches.

\section{Collapsing Nuclear Uncertainties}

As it is indicated in the previous section, the uncertainty information is given in an energy structure. In order to compare libraries, collapsing to one-group is an easy way to analyse the differences among them.

The "sandwich rule", represented by Eq. (1), is used here to go from any energy structure to one-group.

$\Delta^{2}=\omega V \omega^{T}$,

where:

- $\omega=\left(\sigma_{t} \phi_{t}\right)^{-1}\left[\sigma_{1} \phi_{1}, \sigma_{2} \phi_{2 t}, \ldots, \sigma_{n} \phi_{n}\right]$ is the relative cross section vector. $\sigma_{i}$ is the cross-section, $\emptyset_{i}$ is the neutron flux in the energy group i. The sub index $t$ refers to the sum over all the groups.

- $V$ is the relative covariance matrix of the cross sections that are collapsed.

- $\Delta$ is the relative error for one-group.

Before collapsing data, EAF uncertainty values should be divided by a factor of 3 . That is because an uncertainty 
value of three times the experimental uncertainty has been used, under the hypothesis that with this value $99.9 \%$ of the possible values is covered. That represents a 99.9\% confidence limit (if a normal distribution is supposed) $[3,4]$.

\section{Comparison among Libraries using Different Neutron Spectra}

Using the collapsing method showed above, three different neutron spectra will be used to compare uncertainty values.

These spectra are presented in Fig. 1 for each application. The ADS spectrum is the same as that used in [2]. The PWR and DEMO spectra are standard.

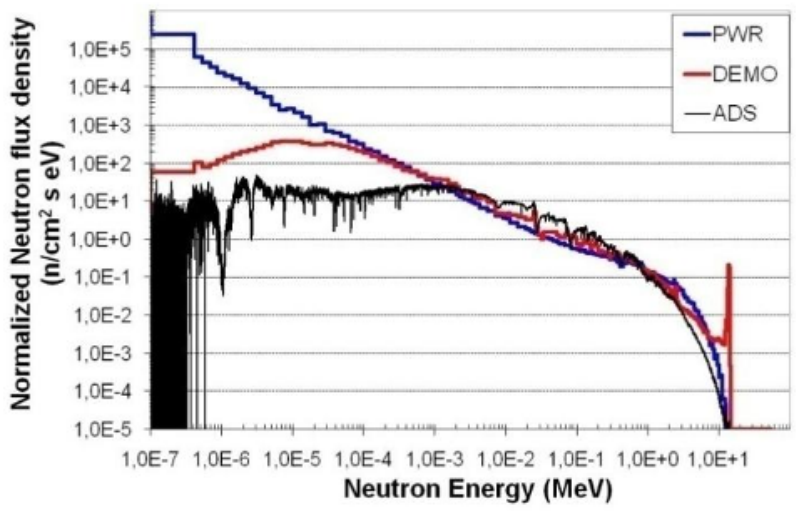

Fig. 1. ADS, PWR and DEMO spectra.

\subsection{Using ADS Neutron Spectrum}

For the ADS spectrum, main transuranic isotopes $\left({ }^{234,235,236,238} \mathrm{U}, \quad{ }^{237} \mathrm{~Np}, \quad{ }^{238,239,240,241} \mathrm{Pu},{ }^{241,242 \mathrm{~m}, 243} \mathrm{Am}\right.$, $\left.242,243,244,245,246,247,248 \mathrm{Cm},{ }^{249} \mathrm{Bk},{ }^{249,250,251,252} \mathrm{Cf}\right)$ are studied for (n,fission), (n,gamma) and (n,gamma-M) reactions. Their collapsed values are compared with target accuracies provided by [2].
Table 1 shows for each reaction the one-group uncertainty for each library and each isotope. The orange cells indicate for which isotopes and reactions target accuracies are available. Then, if any of the values is less than twice times the target value, they are written in bold blue while the others are non-bold.

The results in Table 1 reveal that SCALE-6.0 has no uncertainty information for (n,gamma-M) reactions for any isotope. This library gives the lowest uncertainty values for some isotopes, such as ${ }^{235,238} \mathrm{U}$ and ${ }^{239,241} \mathrm{Pu}$, while EAF-2010 does not reach this accuracy level. EAF2010 shows a tendency of reducing uncertainties when it is compared with EAF-2007. Also, this library gives lower uncertainty values than SCALE-6.0, such as ${ }^{243,246} \mathrm{Cm}$ and ${ }^{249} \mathrm{Cf}$.

Regarding target accuracies, for (n,fission) reaction EAF-2010 achieved two targets: for ${ }^{235} \mathrm{U}$ and ${ }^{241} \mathrm{Pu}$, while SCALE-6.0 achieved it for these two and ${ }^{239} \mathrm{Pu}$. For (n,gamma), SCALE-6.0 is close to achieve it for eight targets while EAF-2010 is close for 11 targets, but in this case, SCALE-6.0 achieved targets for U, Np, Pu and two $\mathrm{Cm}$ while EAF-2010 achieved the targets for $\mathrm{Pu}, \mathrm{Cm}$ and Cf. For the (n,gamma-M) reaction, only EAF files give values, and only EAF-2010 achieved one target value for ${ }^{243} \mathrm{Cm}$.

Fig. 2 presents the uncertainty values for the (n,gamma) reaction of EAF-2007 divided by EAF-2010 values for all isotopes in the libraries. It shows that for most of the isotopes, EAF-2010 has reduced their uncertainty values.

$(n, v)$ EAF-07/EAF-10

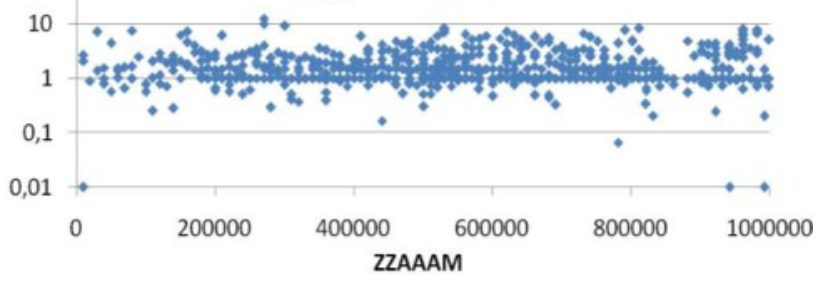

Fig. 2. EAF-2007 uncertainty values divided by those of EAF2010 for the (n,gamma) reaction using the ADS spectrum.

Table 1. Comparison among EAF-2007, EAF-2010 and SCALE-6.0 libraries for main transuranic isotopes and (n,fission), (n,gamma) and (n,gamma-M) reactions using the ADS spectrum.

\begin{tabular}{|c|c|c|c|c|c|c|c|c|c|c|c|c|}
\hline & \multicolumn{4}{|c|}{ (n,fission) } & \multicolumn{4}{|c|}{$(n, y)$} & \multicolumn{4}{|c|}{$(\mathrm{n}, \boldsymbol{\gamma}-\mathrm{M})$} \\
\hline Isotope & EAF2007 & EAF2010 & SCALE6.0 & TARGET & EAF2007 & EAF2010 & SCALE6.0 & TARGET & EAF2007 & EAF2010 & SCALE6.0 & TARGET \\
\hline 2340 & 16.5 & 16.5 & 30.0 & & 38.9 & 26.0 & 6.9 & 7.1 & 38.9 & 26.0 & - & 7.1 \\
\hline $235 \mathrm{U}$ & 12.9 & 5.5 & 0.4 & 4.2 & 11.3 & 3.2 & 21.8 & & - & - & - & \\
\hline $236 \mathrm{U}$ & 15.9 & 15.3 & 27.2 & & 8.9 & 3.2 & 3.1 & & - & - & - & \\
\hline $238 \mathrm{U}$ & 16.6 & 16.6 & 0.5 & & 6.7 & 3.2 & 1.4 & & - & - & - & \\
\hline $237 \mathrm{~Np}$ & 16.7 & 16.4 & 6.6 & & 14.3 & 9.1 & 3.3 & 28 & - & $=$ & - & \\
\hline $238 \mathrm{Pu}$ & 12.4 & 10.1 & 10.6 & 6.4 & 14.5 & 3.7 & 6.6 & 5.2 & - & - & - & \\
\hline 239Pu & 9.6 & 7.9 & 0.4 & 3.4 & 125 & 4.2 & 4.8 & & - & - & - & \\
\hline $240 \mathrm{Pu}$ & 15.8 & 14.7 & 0.6 & & 9.3 & 3.6 & 1.2 & 4.8 & - & - & - & \\
\hline 241Pu & 15.6 & 5.6 & 1.2 & 4.2 & 15.4 & 5.2 & 4.0 & & - & - & - & \\
\hline $242 \mathrm{Pu}$ & 16.5 & 16.5 & 3.4 & & 12.6 & 3.5 & 5.0 & 5.3 & - & $=$ & - & \\
\hline $244 \mathrm{Pu}$ & 16.5 & 16.5 & 19.0 & & 30.4 & 7.4 & 24.9 & & - & - & - & \\
\hline 241Am & 16.6 & 16.6 & 22 & & 15.8 & 16.7 & 4.7 & 28 & 15.8 & 16.7 & - & 29 \\
\hline 242MAm & 16.5 & 5.6 & 9.8 & 2.4 & 32.8 & 13.2 & 14.5 & 6.2 & - & - & - & \\
\hline $243 \mathrm{Am}$ & 16.6 & 16.0 & 5.8 & & 15.3 & 5.0 & 4.5 & & 15.3 & 3.8 & - & 4.1 \\
\hline $242 \mathrm{Cm}$ & 16.5 & 16.5 & 31.9 & & 30.0 & 12.9 & 10.8 & 3.4 & - & - & - & \\
\hline $243 \mathrm{Cm}$ & 16.0 & 5.9 & 19.7 & 3.2 & 32.0 & 5.2 & 14.2 & 7.4 & - & - & - & \\
\hline $244 \mathrm{Cm}$ & 16.4 & 14.8 & 37.0 & & 24.6 & 3.7 & 7.7 & 4.6 & - & - & - & \\
\hline $245 \mathrm{Cm}$ & 9.8 & 11.3 & 20.2 & 4.1 & 32.8 & 4.1 & 9.8 & 5.5 & - & - & - & \\
\hline $246 \mathrm{Cm}$ & 16.6 & 15.2 & 8.0 & & 28.2 & 3.7 & 20.3 & 4.3 & - & - & - & \\
\hline $247 \mathrm{Cm}$ & 16.5 & 16.5 & 11.3 & 4.0 & 32.1 & 7.7 & 20.6 & 5.0 & - & - & - & \\
\hline $248 \mathrm{Cm}$ & 16.2 & 15.3 & 16.2 & & 19.2 & 3.8 & 16.9 & 25 & - & - & - & \\
\hline $249 \mathrm{Bk}$ & 16.6 & 16.6 & 225 & & 31.7 & 8.8 & 23.7 & 3.2 & - & - & - & \\
\hline 249Cf & 16.3 & 5.8 & 19.3 & & 32.4 & 4.8 & 24.5 & 4.3 & - & - & - & \\
\hline $250 \mathrm{Cf}$ & 33.0 & 33.0 & 13.3 & 6.9 & 29.3 & 9.0 & 16.0 & 26 & - & - & - & \\
\hline $251 \mathrm{Cf}$ & 31.6 & 12.9 & 21.9 & 3.7 & 30.0 & 3.9 & 16.6 & 24 & - & - & - & \\
\hline $252 \mathrm{Cf}$ & 15.0 & 10.7 & 6.1 & & 31.2 & 4.0 & 18.1 & & - & - & - & \\
\hline
\end{tabular}


Fig. 3 presents the uncertainty values for the (n,gamma) reaction of SCALE-6.0 divided by EAF-2010 values for all isotopes in the libraries. It shows that for light isotopes, EAF-2010 provides lower uncertainties than SCALE-6.0, but while ZZAAAM increases, the amount of isotopes with high uncertainties on SCALE-6.0 is similar than EAF-2010. The 0.01 values indicate that SCALE-6.0 does not supply uncertainties for these isotopes.

$(n, v)$ SCALE-6.0/EAF-10

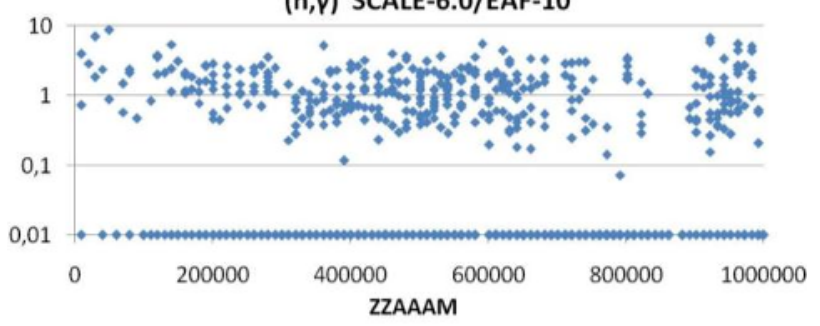

Fig. 3. SCALE-6.0 uncertainty values divided by those of EAF2010 for the (n,gamma) reaction using the ADS spectrum.

Fig. 4 presents EAF-2007 uncertainty values divided by those of EAF-2010 for the (n,fission) reaction. EAF-2010 has reduced their uncertainty values except for ${ }^{242} \mathrm{Am}$.

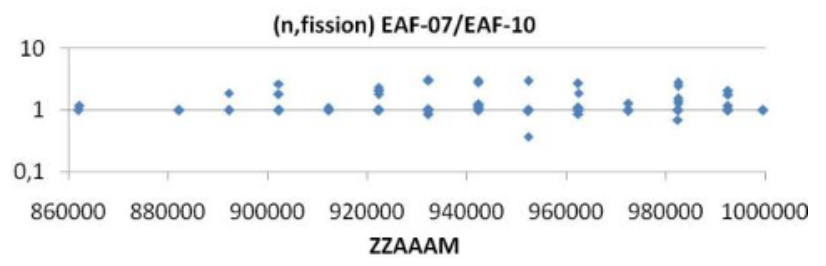

Fig. 4. EAF-2007 uncertainty values divided by those of EAF-

2010 for the $(\mathrm{n}$,fission) reaction using the ADS spectrum.

Fig. 5 shows SCALE-6.0 uncertainty values divided by those of EAF-2010 for the (n,fission) reaction. SCALE6.0 has no uncertainties for some isotopes that have uncertainties on EAF-2010, but SCALE-6.0 gives lower uncertainty values than EAF-2010, especially for ${ }^{238} \mathrm{U}$, ${ }^{239,240} \mathrm{Pu}$. The isotopes with 0.01 values are those for which SCALE-6.0 does not provide uncertainty values.

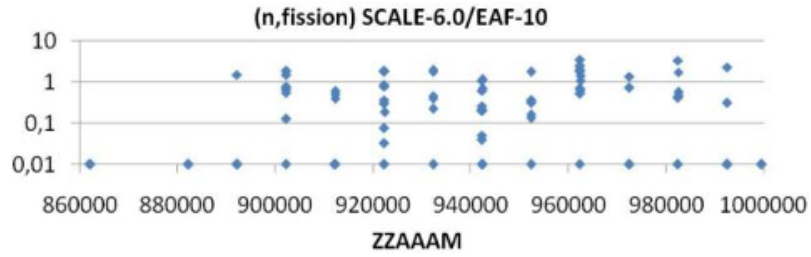

Fig. 5. SCALE-6.0 uncertainty values divided by those of EAF2010 for the (n.fission) reaction using the ADS spectrum.

\subsection{Using PWR Neutron Spectrum}

For high burn-up PWR (100 GWd/kg), Ref. [1] points out several isotopes for which uncertainties should be reduced in order to achieve general target accuracies presented in Table 3.

Table 3. General target accuracies for high burn-up PWR.

\begin{tabular}{|c|c|c|c|}
\hline $\mathrm{k}_{\mathrm{eff}}$ & $\begin{array}{c}\text { Doppler reactivity } \\
\text { coefficient }\end{array}$ & $\begin{array}{c}\text { Burn-up } \\
\Delta \rho\end{array}$ & Transmutation \\
\hline $0.5 \%$ & $10 \%$ & $500 \mathrm{pcm}$ & $5 \%$ \\
\hline
\end{tabular}

Using this information, target accuracies for ${ }^{235,238} \mathrm{U}$, ${ }_{239,240,241,242} \mathrm{Pu}$ and $\mathrm{O}$ are calculated and are compared with the information in EAF-2007 and EAF-2010 in Table 2. It is observed how EAF-2010 has reduced its uncertainties, also inside its energy structure. This Table 2 also shows if EAF files fulfil or not the target accuracies using a colour legend, where green means target achieved, yellow means that not all energy group targets are achieved, and red that no energy group target is achieved. Requirements for the ${ }^{235} \mathrm{U}$ and ${ }^{240,241} \mathrm{Pu}$ for the (n,gamma) reaction are fulfilled with EAF-2010.

When main transuranic isotopes are observed using one-group uncertainties for (n,fission) and (n,gamma)

Table 2. Target accuracies for high burn-up PWR for U, Pu and O.

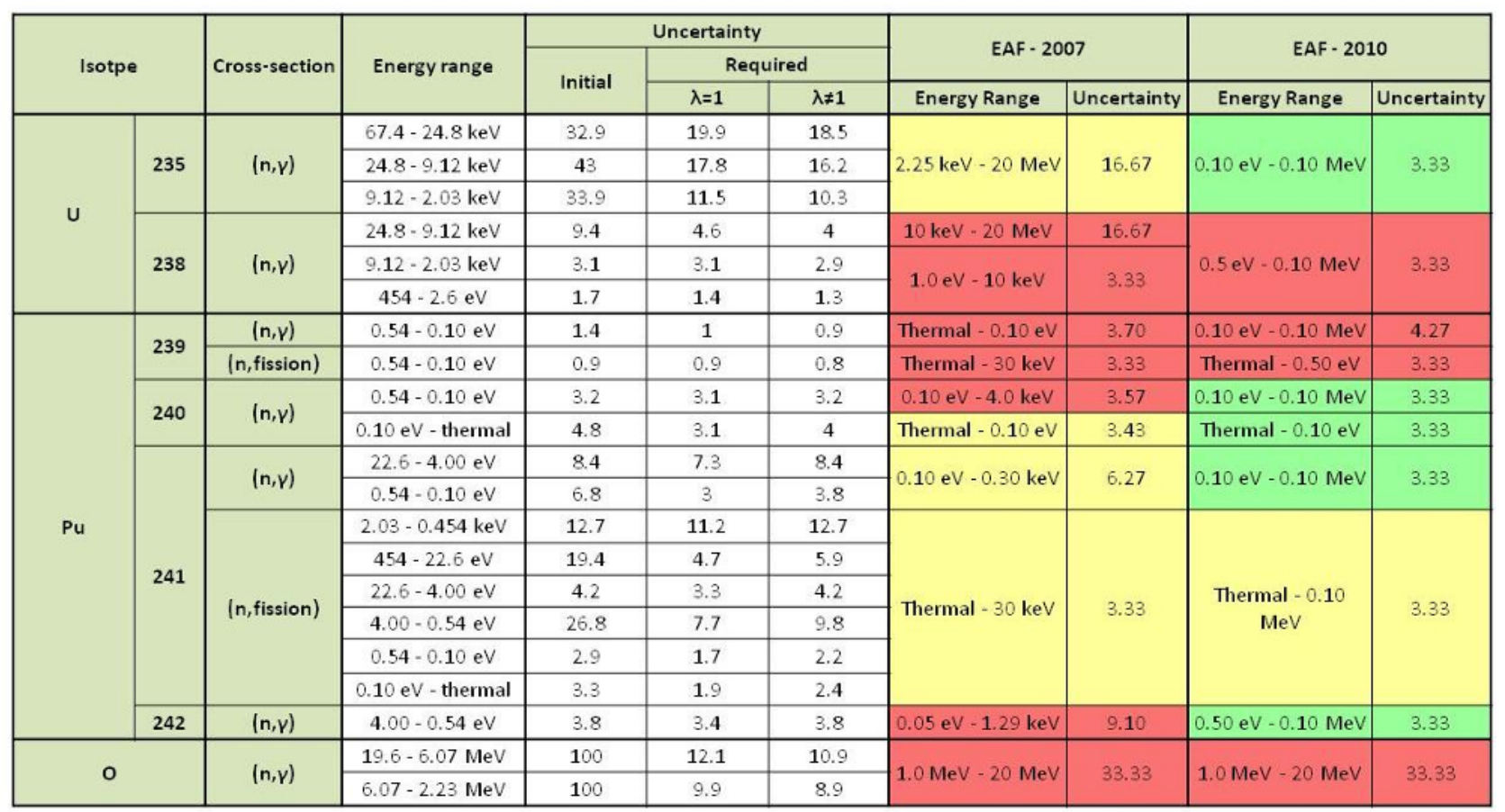


reactions (Table 4), SCALE-6.0 gives lowest uncertainties for 17 isotopes while EAF-2010 does this only for $12\left({ }^{241 \mathrm{~m}} \mathrm{Am},{ }^{243} \mathrm{Cm}\right.$ and ${ }^{249} \mathrm{Cf}$ uncertainty values are very similar using EAF-2010 or SCALE-6.0).

Table 4. One-group uncertainties for main transuranic isotopes for high burn-up PWR.

\begin{tabular}{|c|c|c|c|c|c|c|c|}
\hline \multirow{2}{*}{\multicolumn{2}{|c|}{ ISOTOPE }} & \multicolumn{3}{|c|}{ (n,fission) } & \multicolumn{3}{|c|}{$(n, \gamma)$} \\
\hline & & EAF-2007 & EAF-2010 & SCALE 6.0 & EAF-2007 & EAF-2010 & SCALE 6.0 \\
\hline \multirow{4}{*}{ u } & 234 & 15.86 & 15.84 & 24.82 & 38.87 & 26.03 & 5.73 \\
\hline & 235 & 2.39 & 2.39 & 0.33 & 2.64 & 2.35 & 1.35 \\
\hline & 236 & 11.67 & 12.16 & 19.5 & 3.85 & 3.1 & 2.99 \\
\hline & 238 & 16.65 & 16.65 & 0.52 & 3.15 & 3.17 & 1.38 \\
\hline $\mathrm{Np}$ & 237 & 16.5 & 16.41 & 7 & 5.72 & 7.58 & 2.68 \\
\hline \multirow{6}{*}{$\mathrm{Pu}$} & 238 & 7.23 & 4.86 & 6.01 & 3.73 & 3.07 & 1.79 \\
\hline & 239 & 3.32 & 3.2 & 0.78 & 8.21 & 3.55 & 1.17 \\
\hline & 240 & 14.94 & 14.27 & 2.7 & 3.3 & 3.09 & 1.23 \\
\hline & 241 & 3.3 & 3.32 & 0.87 & 3.9 & 2.39 & 0.94 \\
\hline & 242 & 15.81 & 15.77 & 4.53 & 8.51 & 3.31 & 9.76 \\
\hline & 244 & 16.56 & 16.56 & 21.32 & 23.48 & 4.87 & 35.39 \\
\hline \multirow{3}{*}{ Am } & 241 & 21.34 & 12.44 & 1.66 & 6.38 & 3.89 & 2.5 \\
\hline & $241 \mathrm{M}$ & 3.31 & 3.33 & 3.05 & 22.36 & 10.19 & 23.2 \\
\hline & 243 & 15 & 14.62 & 5.12 & 3.72 & 4.44 & 2.41 \\
\hline \multirow{7}{*}{$\mathrm{Cm}$} & 242 & 16.6 & 10.79 & 32.83 & 19.29 & 9.42 & 12.05 \\
\hline & 243 & 3.94 & 2.56 & 2.71 & 5.94 & 2.35 & 5.58 \\
\hline & 244 & 13.36 & 12.22 & 25.8 & 6.93 & 3.01 & 9.99 \\
\hline & 245 & 3.65 & 5.03 & 2.45 & 14.68 & 2.67 & 4.28 \\
\hline & 246 & 14.6 & 13.67 & 8.37 & 7.99 & 3.15 & 5.63 \\
\hline & 247 & 4.96 & 5.25 & 13.04 & 16.51 & 7.63 & 6.33 \\
\hline & 248 & 12.91 & 13.36 & 16.33 & 10.48 & 3.57 & 5.5 \\
\hline Bk & 249 & 28.84 & 14.56 & 6.47 & 9.52 & 7.74 & 4.96 \\
\hline \multirow{4}{*}{ Cf } & 249 & 7.3 & 2.6 & 1.76 & 3.94 & 2.51 & 4.39 \\
\hline & 250 & 13.81 & 41.36 & 0.6 & 4.85 & 5.93 & 5.91 \\
\hline & 251 & 8.74 & 5.7 & 4.37 & 5.95 & 2.88 & 4.73 \\
\hline & 252 & 11.69 & 4.84 & 11.5 & 12.14 & 2.65 & 5.13 \\
\hline
\end{tabular}

Fig. 6 shows the differences between EAF-2007 and EAF-2010 for the (n,gamma) reaction. A big amount of isotopes have reduced their uncertainties in EAF-2010.

\section{$(n, v)$ EAF-07/EAF-10}

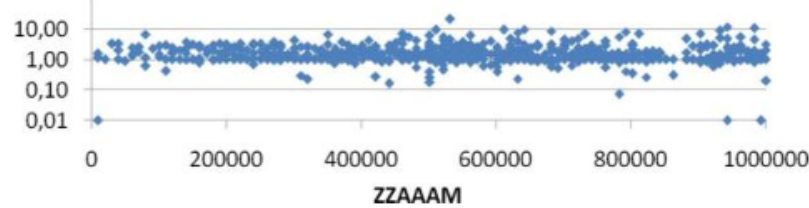

Fig. 6. EAF-2007 uncertainty values divided by those of EAF2010 for the (n,gamma) reaction using the PWR spectrum.

Fig. 7 shows the same trend as for the ADS spectrum when SCALE-6.0 is compared with EAF-2010. SCALE6.0 does not give uncertainty values for $54 \%$ of the isotopes included in the EAF-2010 library. But it provides very low uncertainty values, such as for ${ }^{135} \mathrm{I}$ and ${ }^{79} \mathrm{Se}$.

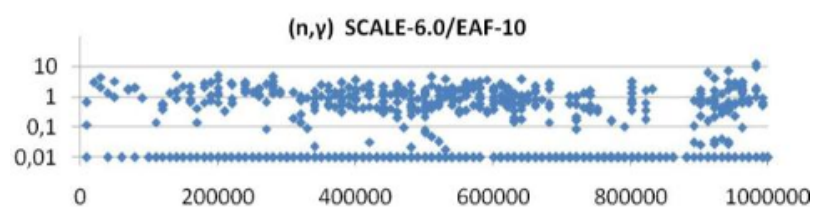

Fig. 7. SCALE-6.0 uncertainty values divided by those of EAF2010 for the (n,gamma) reaction using the PWR spectrum.

Fig. 8 presents EAF-2007 uncertainty values divided by those of EAF-2010 for the (n,fission) reaction. At least $55 \%$ of the isotopes in EAF-2007 have reduced their uncertainties. But not all values have been reduced, such as ${ }^{250} \mathrm{Cf}$.

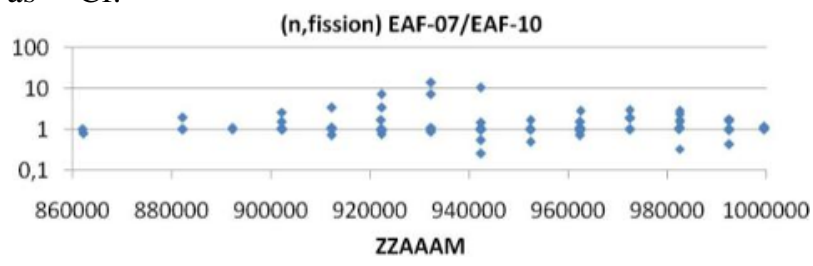

Fig. 8. EAF-2007 uncertainty values divided by those of EAF-

2010 for the (n,fission) reaction using the PWR spectrum.

Fig. 9 shows the differences between SCALE-6.0 and EAF-2010. SCALE-6.0 does not give uncertainties for $42 \%$ of the isotopes included in EAF-2010, but $36 \%$ of the isotopes have lower uncertainties in SCALE-6.0 than in EAF-2010. Also, very low uncertainty values are provided by SCALE-6.0, e.g. for ${ }^{238} \mathrm{U}$ and ${ }^{250} \mathrm{Cf}$.

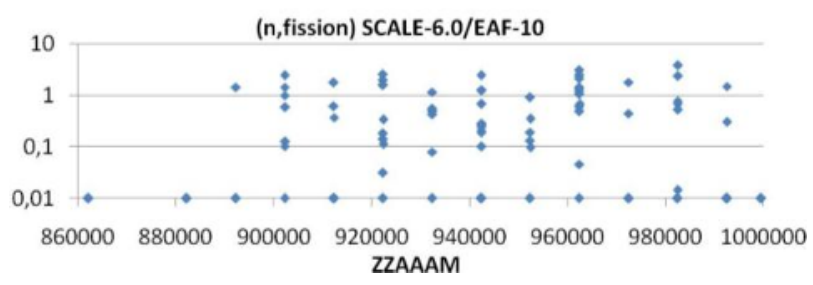

Fig. 9. SCALE-6.0 uncertainty values divided by those of EAF2010 for the (n,fission) reaction using the PWR spectrum.

\subsection{Using DEMO Neutron Spectrum}

For DEMO application, Fig. 10 presents the uncertainty values for EAF-2007, EAF-2010 and SCALE-6.0 for all isotopes in the libraries in order to show the bulk of the uncertainties. It shows that the bulk of uncertainties is between $3 \%$ and $40 \%$. SCALE-6.0 provides the lowest uncertainties but lacks in giving uncertainties for $54 \%$ of the isotopes included in EAF files (values with $0.1 \%$ error).

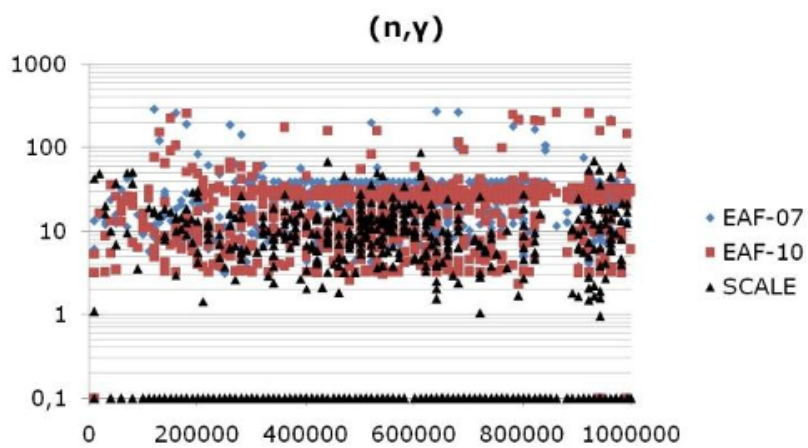

Fig. 10. All uncertainty values for all isotopes included in EAF2007, EAF-2010 and SCALE-6.0 for the (n,gamma) reaction using the DEMO spectrum.

When the (n,p) reaction is observed in Fig. 11, EAF2010 exhibits increased uncertainties for this reaction compared to EAF-2007. This trend is also observed using other spectra (ADS and PWR). Fig. 12 shows the differences between SCALE-6.0 and EAF-2010. SCALE6.0 lacks not only in giving uncertainty, but also in giving lower uncertainties than EAF-2010 for the (n,p) reaction. 


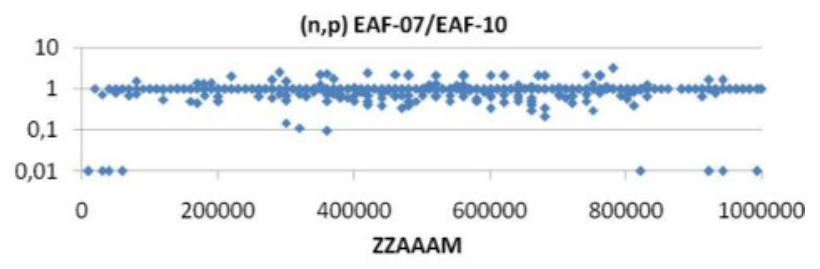

Fig. 11. EAF-2007 uncertainty values divided by those of EAF2010 for the $(n, p)$ reaction using the DEMO spectrum.

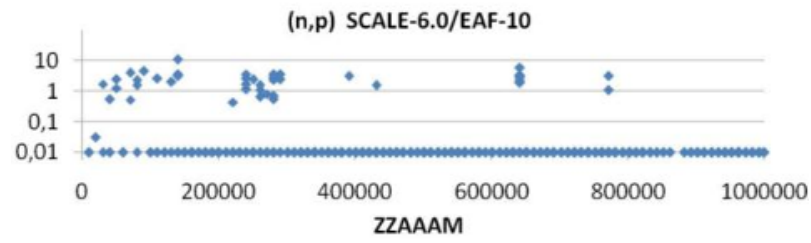

Fig. 12 SCALE-6.0 uncertainty values divided by those of EAF2010 for the $(n, p)$ reaction using the DEMO spectrum.

\section{Analysis of the Effect of Neutron Spectra}

Table 5 gives the comparison of the uncertainties for each library (EAF-2010 and SCALE-6.0) when different neutron spectra are used. In this case, only (n,gamma) and (n,fission) are studied. For one reaction, each library is collapsed to one-group using different neutron spectra, and their results are compared.

The colour legend for Table 5 is: green for the lowest uncertainty value among neutron spectra, yellow for the middle value and red for the highest.

For the (n,gamma) reaction, EAF-2010 gives the lowest values when the PWR neutron spectrum is used, while ADS provokes the highest uncertainties. However DEMO gives lower uncertainties than ADS but not lower than for PWR. SCALE-6.0 gives the highest uncertainty values for ADS, but PWR and DEMO obtain almost the lowest values (PWR obtains more green cells than DEMO, but the differences are small). It is important to remark that when SCALE-6.0 is used for the (n,gamma) reaction, the uncertainty value obtained for some of the main transuranic isotopes can double the values obtained using PWR or DEMO spectra.

For the (n,fission) reaction, EAF-2010 gives the lowest values when the PWR spectrum is used; the highest values for ADS; DEMO spectrum does not provide low values for the (n,gamma) reaction, and they are as high as for ADS for some isotopes such as ${ }^{236} \mathrm{U}$. SCALE-6.0 follows the same trend for the (n,gamma) reaction, the highest values are reached when the ADS spectrum is used. This time, PWR and DEMO have also the same amount of highest values. DEMO gives the highest values for ${ }^{241,243} \mathrm{Am}$, while PWR gives the highest for ${ }^{237} \mathrm{~Np}$.

The ADS spectrum reaches the highest uncertainties for main transuranic isotopes when (n,gamma) and (n,fission) reactions are studied for both libraries: EAF2010 and SCALE-6.0. PWR spectrum provokes the lowest uncertainties when EAF-2010 is used. But when SCALE-6.0 is used, it is easy to distinguish which spectrum reaches the lowest uncertainties, DEMO or PWR.

\section{Conclusions}

The comparison among libraries shows how different they are. It shows the changes of EAF-2010 from EAF2007, where many isotopes have reduced their uncertainties for (n,gamma) and (n,fission) reactions; but not for $(n, p)$ reactions, where many uncertainties remain as before or have been increased. This trend is observed for all neutron spectra. The main reason behind this

Table 5. Comparison between EAF-2010 and SCALE-6.0 for (n,gamma) and (n,fission) using PWR, ADS and DEMO spectra for main transuranic isotopes.

\begin{tabular}{|c|c|c|c|c|c|c|c|c|c|c|c|c|c|}
\hline & & \multicolumn{6}{|c|}{$(\mathbf{n}$, gamma $)$} & \multicolumn{6}{|c|}{ (n,fission) } \\
\hline & & \multicolumn{3}{|c|}{ EAF-2010 } & \multicolumn{3}{|c|}{ SCALE 6.0 } & \multicolumn{3}{|c|}{ EAF-2010 } & \multicolumn{3}{|c|}{ SCALE 6.0 } \\
\hline \multicolumn{2}{|c|}{ ISOTOPE } & $P W R$ & $A D S$ & DEMO & $P W R$ & $A D S$ & DEMO & $P W R$ & $A D S$ & DEMO & $P W R$ & $A D S$ & DEMO \\
\hline \multirow{4}{*}{$\mathbf{U}$} & 234 & 26.03 & 26.03 & 26.03 & 5.73 & 6.93 & 4.95 & 15.84 & 16.47 & 16.43 & 24.82 & 29.99 & 15.37 \\
\hline & 235 & 2.35 & 3.23 & 3.2 & 1.35 & 21.8 & 7.32 & 2.39 & 5.5 & 4.7 & 0.33 & 0.41 & 0.3 \\
\hline & 236 & 3.1 & 3.2 & 3.24 & 2.99 & 3.11 & 2.07 & 12.16 & 15.31 & 15.63 & 19.5 & 27.16 & 11.42 \\
\hline & 238 & 3.17 & 3.17 & 3.27 & 1.38 & 1.44 & 1.49 & 16.65 & 16.61 & 16.66 & 0.52 & 0.54 & 0.55 \\
\hline Np & 237 & 7.58 & 9.13 & 9.79 & 2.68 & 3.3 & 2.21 & 16.41 & 16.39 & 16.55 & 7 & 6.55 & 3.81 \\
\hline \multirow{6}{*}{ Pu } & 238 & 3.07 & 3.69 & 3.27 & 1.79 & 6.63 & 3.84 & 4.86 & 10.09 & 11.21 & 6.01 & 10.55 & 10.75 \\
\hline & 239 & 3.55 & 4.21 & 4.18 & 1.17 & 4.86 & 1.59 & 3.2 & 7.87 & 6.48 & 0.78 & 0.4 & 0.58 \\
\hline & 240 & 3.09 & 3.62 & 3.28 & 1.23 & 1.2 & 0.96 & 14.27 & 14.68 & 15.87 & 2.7 & 0.57 & 0.59 \\
\hline & 241 & 2.39 & 5.22 & 3.27 & 0.94 & 4 & 1.9 & 3.32 & 5.64 & 4.3 & 0.87 & 1.23 & 0.75 \\
\hline & 242 & 3.31 & 3.51 & 3.27 & 9.76 & 5 & 6.36 & 15.77 & 16.46 & 16.52 & 4.53 & 3.43 & 3.61 \\
\hline & 244 & 4.87 & 7.36 & 5.64 & 35.39 & 24.88 & 35.87 & 16.56 & 16.47 & 16.59 & 21.32 & 18.96 & 17.29 \\
\hline \multirow{3}{*}{ Am } & 241 & 3.89 & 16.65 & 16.55 & 2.5 & 4.67 & 4.08 & 12.44 & 16.62 & 16.4 & 1.66 & 2.19 & 2.71 \\
\hline & 241M & 10.19 & 13.18 & 10.48 & 23.2 & 14.66 & 12.4 & 3.33 & 5.59 & 4.28 & 3.05 & 9.88 & 7.28 \\
\hline & 243 & 4.44 & 4.98 & 4.71 & 2.41 & 4.48 & 2.71 & 14.62 & 15.95 & 16.29 & 5.12 & 5.76 & 9.67 \\
\hline \multirow{7}{*}{$\mathrm{Cm}$} & 242 & 9.42 & 12.86 & 13.65 & 12.05 & 10.8 & 6.25 & 10.79 & 16.51 & 15.66 & 32.83 & 31.85 & 24.37 \\
\hline & 243 & 2.35 & 5.21 & 3.56 & 5.58 & 14.24 & 10.39 & 2.56 & 5.91 & 4.68 & 2.71 & 19.72 & 9.03 \\
\hline & 244 & 3.01 & 3.72 & 3.27 & 9.99 & 7.72 & 7.18 & 12.22 & 14.82 & 15.39 & 25.8 & 37.01 & 21.33 \\
\hline & 245 & 2.67 & 4.13 & 3.35 & 4.28 & 9.83 & 8.35 & 5.03 & 11.33 & 12.56 & 2.45 & 20.18 & 9.45 \\
\hline & 246 & 3.15 & 3.7 & 3.29 & 5.63 & 20.32 & 8.11 & 13.67 & 15.24 & 15.99 & 8.37 & 8.01 & 8.58 \\
\hline & 247 & 7.63 & 7.67 & 8.17 & 6.33 & 20.59 & 7.13 & 5.25 & 16.46 & 14.44 & 13.04 & 11.3 & 11.42 \\
\hline & 248 & 3.57 & 3.79 & 3.68 & 5.5 & 16.85 & 5.79 & 13.36 & 15.28 & 15.33 & 16.33 & 16.17 & 16.11 \\
\hline Bk & 249 & 7.74 & 8.82 & 8.86 & 4.96 & 23.99 & 9.49 & 14.56 & 16.61 & 16.44 & 6.47 & 22.5 & 20.02 \\
\hline \multirow{4}{*}{ Cf } & 249 & 2.51 & 4.8 & 3.73 & 4.39 & 24.59 & 13.35 & 2.6 & 5.83 & 4.79 & 1.76 & 19.35 & 7.31 \\
\hline & 250 & 5.93 & 8.97 & 9.72 & 5.91 & 16.06 & 4.65 & 41.36 & 32.98 & 30.73 & 0.6 & 13.32 & 12.85 \\
\hline & 251 & 2.88 & 3.85 & 3.22 & 4.73 & 16.89 & 3.99 & 5.7 & 12.92 & 9.5 & 4.37 & 22.02 & 9.23 \\
\hline & 252 & 2.65 & 4.03 & 3.8 & 5.13 & 18.11 & 8.01 & 4.84 & 10.68 & 9.74 & 11.5 & 6.11 & 12.54 \\
\hline
\end{tabular}


reduction is that EAF-2010 has included more experimental data, and performed a review of systematic errors included in the library.

For SCALE-6.0, it can be observed that it lacks in giving uncertainties for many isotopes that EAF files give, but for some isotopes such as ${ }^{235} \mathrm{U}$ and ${ }^{239,241} \mathrm{Pu}$ SCALE-6.0 provides very low uncertainties. For (n,gamma) reactions, it usually gives higher values than EAF-2010 for low atomic mass isotopes, but this trend changes when the atomic mass increases. For the (n,fission) reaction, SCALE-6.0 gives smaller values than EAF-2010 for almost all fissile nuclides.

When target accuracies for ADS are compared with uncertainty values in EAF-2010 and SCALE-6.0, it can be observed that SCALE-6.0 and EAF-2010 reach almost the same targets for the (n,gamma) reaction; but for the (n,fission) reaction, EAF-2010 reaches more targets than SCALE-6.0. Target accuracies for high burn-up PWR are achieved by EAF-2010 but not by EAF-2007.

When the effect of neutron spectra is analysed, the ADS spectrum provokes the highest uncertainties for (n,fission) and (n,gamma) reactions for both libraries, EAF-2010 and SCALE-6.0.

Differences between libraries show that uncertainty information is not complete and well-known. SCALE-6.0 provides very low uncertainty values, sometimes smaller than EAF-2010. However, both libraries share the same experiment information or at least almost, so that, similar results should be achieved but not for all. The main reason is that throughout evaluation stage of a library neither all experiments are considered nor have the same relevance.

From a user point of view, choosing which library should be used would be a problem. This is because there would be a need of data about several isotopes whose information is only included in one library which is not the main library. If both libraries were used, which uncertainty data should be used when the uncertainty information is found twice? Is it possible to mix this uncertainty data or uncertainty data from one library and mean values from another? One solution would be what SCALE uncertainty library [5] says: to use relative (\%) uncertainty information just in order to apply it to different libraries. This method is not the best, but because uncertainty libraries are not complete and wellknown, it is the best thing users of nuclear data libraries and uncertainties can do till more accurate and complete libraries are released. It is better to try to quantify the final uncertainty taking into account all possible uncertainties than to neglect uncertainties without knowing their error contribution.

This work is partially supported by FP7-EURATOM-FISSION2009:Project ANDES/249671 and by the Spanish Science and Innovation Ministry with the FPU Program for teaching and researching formation under grant AP2009-1801 for the first author.

\section{References}

1. M. Salvatores et al. OECD/NEA WPEC Subgroup 26 Final Report: Uncertainty and Target Accuracy Assessment for Innovative Systems Using Recent Covariance Data Evaluations (Report NEA/WPEC26, Paris 2008)

2. N. García-Herranz , O. Cabellos , F. ÁlvarezVelarde, J. Sanz , E.M. González-Romero , J. Juan, Annals of Nuclear Energy 37, (2010) 1570

3. R. A. Forrest, J. Kopecky and J.-Ch. Sublet, The European Activation File: EAF-2007 neutroninduced cross section library (UKAEA, FUS 535, 2007)

4. J.-Ch. Sublet, L. W. Packer, J. Kopecky, R. A. Forrest, A. J. Koning and D. A. Rochman, The European Activation File: EAF-2010 neutroninduced cross section library (CCFE-R(10) 05, 2010)

5. M. L. Williams, D. Wiarda, G. Arbanas, B. L. Broadhead, Scale Nuclear Data Covariance Library (ORNL/TM-2005/39, version 6, vol. III, Sect. M19, Oak Ridge 2009) 